\title{
Detection of organ bacterial load in quails
}

\author{
M.A. Hamad, A.M. Al-Aalim, S.Y.A. Al-Dabbagh and H.H. Ali
}

Department of Microbiology, College of Veterinary Medicine, University of Mosul, Mosul, Iraq

\begin{abstract}
A total of thirty normally quail birds were bought from local market in Mosul city in the period from September-October 2011. Quail birds were slaughtered and samples taken aseptically from internal organs of each bird for bacteriological investigation. The result showed isolation of 203 bacterial isolates from different organs of quail birds. The isolates ranged from Corynebacterium spp. $29.6 \%$ (60 isolates) as a high percent, then E. coli 18.2\% (37 isolates), Staphylococcus aureus $16.3 \%$ (33 isolates), Bacillus spp. 14.8\% (30 isolates), Enterococcus faecalis 9.9\% (20 isolates), Klebsiella pneumoniae 6.4\% (13 isolates), Proteus spp. 1.9\% (4 isolates), Pasteurella multocida 1.9\% (4 isolates) and Coagulase -vestaphyloco-ccus 1\% (2 isolates). This study showedCorynebacterium spp. and E. coli were dominant bacteria in the internal organs of quail birds. Many studies reported that quail birdswere resistant to many bacterial diseases, so that these birds may act asmechanical transporting for different bacterial species to humans and animals with the risky of transporting of resistance bacterial species for many antibiotics.
\end{abstract}

Keywords: Wild birds; Bacterial isolates; Internal organs.

Available online at http://www.vetmedmosul.org/ijvs

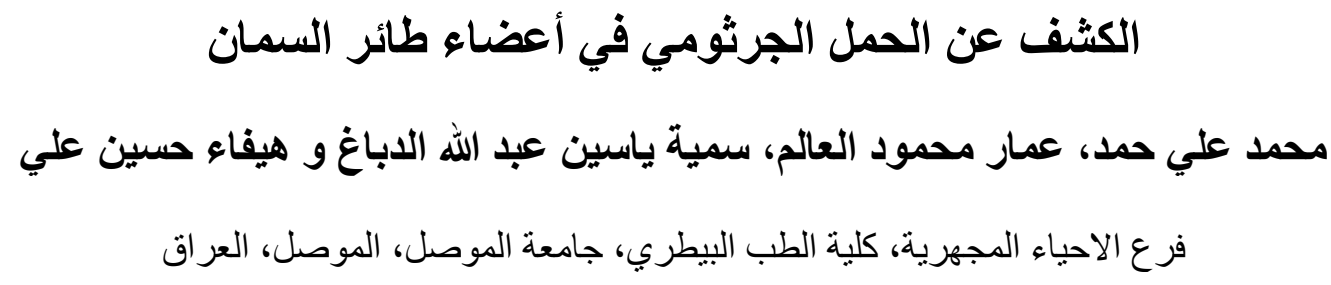

الخلاصة

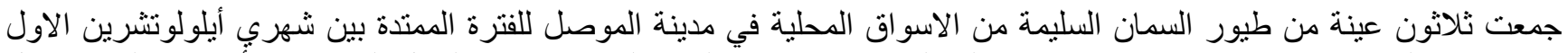

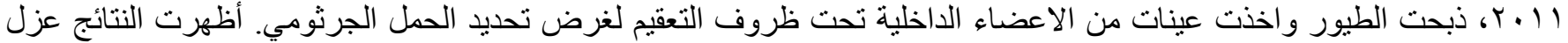

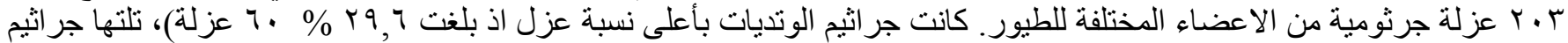

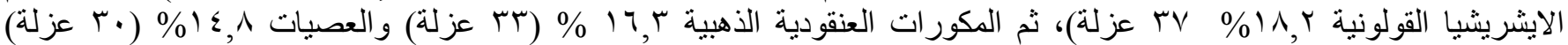

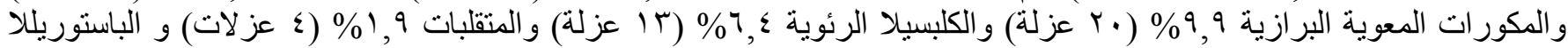

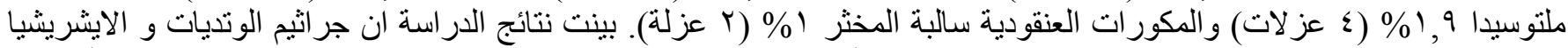

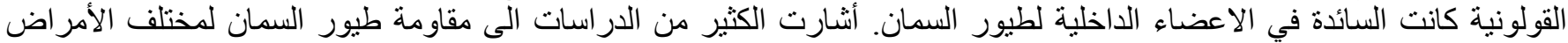
الجرثومية، لذا فإن هذه الطيور قد تلعب دور الناقل الميكانيكي لمعظم الانواع الجرثومية للحيوان والانسان مع خطورة نقلها للجر اثثيم

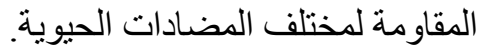

\section{Introduction}

Japanese quails are members of the pheasant family (phasianidae) $(1,2)$. Japan is the native place of this species of quail which was domesticated as long ago as the 12th century, at the beginning of this century these birds have been bred in large numbers for dual-purpose of meat and eggs production $(1,3,4)$. Quail meat in some countries considered as a good food for all ages due to its high meat yield, little shrinkage during cooking, fast cooking and serving and also due to their delicacy and low level of cholesterol. In addition, quail meat is tender and fortified with nutrients $(1,3,5,6)$. Because it is a perfect source of vitamin B6, niacin, thiamin, pantothenic acid and 
riboflavin, so quail meat favorite more than other species of poultry meat $(7,8)$. Japanese quails is extensively distributed in several countries around the world and in some European countries (like France, Italy, Spain and Greece) considerable quantities of quail meat are used for human consumptiondue to their easily adapt to commercial management conditions, with good performance in term of meat and egg produc-tion $(3,7,8)$. In Saudi Arabia and Egypt, quail farms start gaining popularity because their less feed requirements and space $(6,8)$. In our country particularly in Mosul city many quail farms were established and today a considerable numbers of live quail birds are sell in local birds markets for human consumption, so the present study aimed to detect the bacterial types that may be found in the organs of these birds.

\section{Materials and methods}

\section{Specimens}

A total of thirty normally quail birdswere bought from local birds markets in Mosul city and transported directly to laboratory of Microbiology in college of veterinary medicine, then slaughtered and samples taken aseptically from internal organs of each bird that included liver, lung, gizzard and intestine. The samples were put in sterile nutrient broths and incubated at $37^{\circ} \mathrm{C}$ for 24 hours (9).

\section{Culturing}

Each broth was inoculated on three media included nutrient agar, sheep blood agar and MacConkeyagar and incubated aerobically at $37^{\circ} \mathrm{C}$ for $24-48$ hours $(10,11)$.

\section{Identification of Bacterial isolates}

Purification was done and colonial characteristics and blood hemolysis were studied. After that smears from specific colonies were prepared and stained by Gram's stain to study the shape, arrangement and staining reaction (11). Alsosome selective media were used like mannitol salt agar for staphylococci growth, Edward's medium for streptococci, Hoyle's medium for Corynebacterium and MacConkey agar for enterobacteriaceae. Biochemical tests were applied for each specific bacterial isolates and included catalase, oxidase, indole production, methyl red, VP, citrate utilization, gelatin hydrolysis, urease, triple sugar iron, nitrate reduction $(12,13)$.

\section{Results}

Results of the study revealed isolation of numerous bacterial types from different organs of quail birds that included 203 isolates from different organs, which involved Corynebacterium spp.in a high percent $29.6 \%$ (60 isolates), then E. coli 18.2\% (37 isolates), staphylococcus aureus $16.3 \%$ (33 isolates), Bacillus spp. $14.8 \%$ (30 isolates),
Enterococcus faecalis $9.9 \% \quad(20$ isolates), Klebsiella pneumoniae $6.4 \%$ (13 isolates), Proteus spp. $1.9 \%$ (4 isolates), Pasteurella multocida $1.9 \%$ (4 isolates) and Coagulase-vestaphylococcus $1 \% \quad(2$ isolates). These bacterial types classified according to the bird organs as following:

\section{Liver specimens}

Twenty one liver specimens were positive for bacterial isolation $(70 \%)$. Six bacterial types (37 isolates) were isolated from specimens of livers (Table 1). Corynebacterium spp. appeared in a high percent between the total isolates from liver, and also isolated purely more than other bacterial types (Table 2).

Table (1): Isolated bacterial types from livers' specimens.

\begin{tabular}{lcc}
\hline Bacterial type & $\begin{array}{c}\text { Numbers of } \\
\text { isolates }\end{array}$ & $\begin{array}{c}\text { Percentage } \\
\%\end{array}$ \\
\hline Corynebacterium spp. & 13 & 35.14 \\
E. coli & 9 & 24.32 \\
Bacillus spp. & 8 & 21.62 \\
Staphylococcus aureus & 4 & 10.81 \\
Klebsiella pneumoniae & 1 & 2.70 \\
Enterococcus faecalis & 2 & 5.41 \\
\hline Total & 37 & $100 \%$ \\
\hline
\end{tabular}

Table (2): Mixed and pure bacterial types isolated from liver specimens.

\begin{tabular}{clc}
\hline $\begin{array}{c}\text { Numbers of } \\
\text { liver specimens }\end{array}$ & Bacterial types & $\begin{array}{c}\text { Percentage } \\
\%\end{array}$ \\
\hline 6 & Corynebacterium spp. & 28.57 \\
3 & E. coli & 14.29 \\
4 & $\begin{array}{l}\text { Coryne. + Bacillus } \\
\text { Coryne. + Staph. }\end{array}$ & 19.05 \\
3 & $\begin{array}{l}\text { aureus + E. coli } \\
\text { Bacillus spp. }+ \text { E. coli } \\
\text { +Enterococcus }\end{array}$ & 14.29 \\
1 & $\begin{array}{l}\text { Bacillus + E. coli }+ \\
\text { Staph. aureus }\end{array}$ & 4.76 \\
1 & Bacillus + E. coli & 4.76 \\
1 & Klebsiella + & 4.76 \\
1 & Enterococcus & 4.76 \\
1 & Bacillus spp. & 4.76 \\
\hline 21 & Total & $100 \%$ \\
\hline
\end{tabular}

Lung specimens

All lung specimens were positive for bacterial isolation. Eight bacterial types (62 isolates) were isolated from specimens of lungs (Table 3). Corynebacterium spp. appeared predominant bacterial type from lungs as a pure or 
mixed culture, follow-ed by Staphylococcus aureus (Table $4)$.

Table (3): Isolated bacterial types from lung specimens.

\begin{tabular}{lcc}
\hline Bacterial type & $\begin{array}{c}\text { Numbers of } \\
\text { isolates }\end{array}$ & $\begin{array}{c}\text { Percentage } \\
\%\end{array}$ \\
\hline Corynebacterium spp. & 20 & 32.25 \\
Staphylococcus aureus & 13 & 20.98 \\
E. coli & 9 & 14.52 \\
Klebsiella pneumoniae & 6 & 9.67 \\
Bacillus spp. & 6 & 9.67 \\
Pasteurella multocida & 4 & 6.45 \\
Enterococcus faecalis & 2 & 3.23 \\
Coagulase-ve Staph. & 2 & 3.23 \\
\hline Total & 62 & $100 \%$ \\
\hline
\end{tabular}

Table (4): Mixed and pure bacterial types isolated from lung specimens.

\begin{tabular}{|c|c|c|}
\hline $\begin{array}{l}\text { Numbers of lung } \\
\text { specimens }\end{array}$ & Bacterial types & $\begin{array}{c}\text { Percentage } \\
\%\end{array}$ \\
\hline 7 & Coryne. + Staph. aureus & 23.33 \\
\hline 5 & Corynebacterium spp. & 16.67 \\
\hline 4 & $\begin{array}{l}\text { Staphylococcus aureus } \\
\text { E. coli+ Klebsiella }\end{array}$ & 13.33 \\
\hline 4 & $\begin{array}{l}\text { pneumonia + Bacillus } \\
\text { spp. }\end{array}$ & 13.33 \\
\hline 2 & $\begin{array}{l}\text { Coryne. }+ \\
\text { Enterococcus }+ \text { E. coli } \\
\text { Coryne. }+ \text { E. coli }+\end{array}$ & 6.67 \\
\hline 2 & $\begin{array}{l}\text { Coagulase- } \\
\text { Staphylococcus }\end{array}$ & 6.67 \\
\hline 2 & $\begin{array}{l}\text { Pasteurellamultocida + } \\
\text { Coryne. } \\
\text { Pasteurella multocida }+\end{array}$ & 6.67 \\
\hline 2 & $\begin{array}{l}\text { Klebsiella pneumonia }+ \\
\text { Bacillus spp. }\end{array}$ & 6.67 \\
\hline 1 & $\begin{array}{l}\text { Coryne. }+ \text { Staph } . \\
\text { aureus }+ \text { Proteus }\end{array}$ & 3.33 \\
\hline 1 & $\begin{array}{l}\text { Coryne. }+ \text { E. coli }+ \\
\text { Staph. aureus }\end{array}$ & 3.33 \\
\hline 30 & Total & 100 \\
\hline
\end{tabular}

\section{Gizzards specimens}

In gizzard specimens six bacterial types (47 isolates) were isolated and the Gram positive bacteria were dominant, particularly Bacillus spp. and Staphylococcus aureus (table 5). More frequently mixed isolation from these specimens included Bacillus spp. with Enterococcus faecalis (table 6).
Table (5): Isolated bacterial types from gizzards specimens.

\begin{tabular}{lcc}
\hline Bacterial type & $\begin{array}{c}\text { Numbers of } \\
\text { isolates }\end{array}$ & $\begin{array}{c}\text { Percentage } \\
\%\end{array}$ \\
\hline Bacillus spp. & 13 & 27.65 \\
Staphylococcus aureus & 12 & 25.53 \\
Corynebacterium spp. & 9 & 19.15 \\
Enterococcus faecalis & 7 & 14.90 \\
E. coli & 4 & 8.51 \\
Klebsiella pneumoniae & 2 & 4.26 \\
\hline Total & 47 & $100 \%$ \\
\hline
\end{tabular}

Table (6): Mixed and pure bacterial types isolated from gizzards specimens.

\begin{tabular}{|c|c|c|}
\hline $\begin{array}{l}\text { Numbers of } \\
\text { gizzards } \\
\text { specimens }\end{array}$ & Bacterial types & $\begin{array}{c}\text { Percentage } \\
\%\end{array}$ \\
\hline 5 & Corynebacterium spp. & 16.67 \\
\hline 5 & $\begin{array}{l}\text { Bacillus spp. }{ }^{+} \\
\text {Enterococcus faecalis }\end{array}$ & 16.67 \\
\hline 4 & Bacillus spp. & 13.33 \\
\hline 4 & Staphylococcus aureus & 13.33 \\
\hline 4 & Staph. aureus+Bacillusspp. & 13.33 \\
\hline 2 & E. coli & 6.67 \\
\hline 2 & $\begin{array}{l}\text { Klebsiella pneumonia }+ \\
\text { Enterococcus faecalis }\end{array}$ & 6.67 \\
\hline 2 & Coryne. + Staph. aureus & 6.67 \\
\hline 1 & $\begin{array}{l}\text { Coryne. + E. coli+ Staph. } \\
\text { aureus }\end{array}$ & 3.33 \\
\hline 1 & $\begin{array}{l}\text { Coryne. }+ \text { E. coli }+ \text { Staph. } \\
\text { aureus }\end{array}$ & 3.33 \\
\hline 30 & Total & 100 \\
\hline
\end{tabular}

\section{Intestinal specimens}

Between seven bacterial types (included 57 isolates) isolated from intestines of quail birds, Corynebacterium spp. and E. coli represented in a high percent (table 7), also these two types isolated together more frequently as a mixed culture (table 8).

Table (7): Isolated bacterial types from intestines specimens.

\begin{tabular}{lcc}
\hline Bacterial type & $\begin{array}{c}\text { Numbers of } \\
\text { isolates }\end{array}$ & $\begin{array}{c}\text { Percentage } \\
\%\end{array}$ \\
\hline Corynebacterium spp. & 18 & 31.58 \\
E. coli & 15 & 26.32 \\
Enterococcus faecalis & 9 & 15.79 \\
Staphylococcus aureus & 4 & 7.02 \\
Klebsiella pneumoniae & 4 & 7.02 \\
Proteus spp. & 4 & 7.02 \\
Bacillus spp. & 3 & 5.25 \\
\hline Total & 57 & $100 \%$ \\
\hline
\end{tabular}


Table (8): Mixed and pure bacterial types isolated from intestines specimens.

\begin{tabular}{clc}
\hline $\begin{array}{l}\text { Numbers } \\
\text { intestines } \\
\text { specimens }\end{array}$ & Bacterial types & $\begin{array}{l}\text { Percentage } \\
\%\end{array}$ \\
\hline 5 & Corynebacterium spp. & 16.67 \\
5 & $\begin{array}{l}\text { E. coli } \\
\text { Coryne. }+ \text { E. coli }\end{array}$ & 16.67 \\
5 & $\begin{array}{l}\text { E. coli + Enterococcus } \\
\text { faecalis }\end{array}$ & 16.67 \\
4 & $\begin{array}{l}\text { Coryne. }+ \text { Enterococcus } \\
\text { faecalis + Proteus spp. }\end{array}$ & 10 \\
3 & $\begin{array}{l}\text { Coryne. }+ \text { Staph. aureus } \\
+ \text { Bacillus spp. }\end{array}$ & 10 \\
3 & $\begin{array}{l}\text { Klebsiella pneumoniae+ } \\
\text { Enterococcus faecalis }\end{array}$ & 6.68 \\
2 & $\begin{array}{l}\text { Coryne. }+ \text { Klebsiella } \\
\text { pneumoniae }\end{array}$ & 6.68 \\
2 & $\begin{array}{l}\text { Proteus + E. coli + Staph. } \\
\text { aureus }\end{array}$ & 3.33 \\
\hline 30 & Total & 100 \\
\hline
\end{tabular}

\section{Discussion}

According to the results of this study many bacterial types were isolated from different organs of quail birds involved Corynebacterium spp., E. coli, Staphylococcusaureus, Klebsiellapneumoniae, Enterococcus faecalis, Bacillus spp., Prot-eus spp., Pasteurellamultocida and Coagulase-veStaphylococcus. These results were agreed to the results of previous studies about the isolation of same bacterial types from quail, but differed from them in rates of isolates $(8,14-16)$. The differences between theresults could be attributed to the variations in climate and environment of husbandry regions especially temperatures variation that effect on the bacterial growth $(4,17)$.

Results of cultured liver samples revealed isolation of six bacterial types (Table 1) includedCorynebacterium spp., E. coli, Bacillus spp., Staphylococcus aureus, Klebsiella pneumonia and Enterococcus faecalis. Many studies referred to the isolation of one or more of these bacterial species from livers particularly E. coli, Enterococci and Staphylococci (16,18-20), while Corynebacterium spp. isolationwas not referred previously; and this will confirm the environmental changes (17). The bacterial types isolated from liver specimens were similar to those isolated from intestine and this conclude that livers bacteria might passed from intestine to liver or may reach the liver by extension from adjacent air sacs or from less frequently, by extension up the biliary tree (21).
Eight bacterial types (62 isolates) were isolated from specimens of lungs (Table 3). Corynebacterium spp. appeared predominant bacterial type from lungs as a pure or mixed culture, followed by Staphylococcus aureus, E. coli, Klebsiellapneumonia, Bacillus spp., Pasteurellamultocida, Enterococcus faecalis, Coagulase -veStaphylococci (Table 4). Many researches were observed isolation of these bacterial species from lungs of diseased quails $(16,18,20,22,23)$. Gizzard specimens were appeared positive for bacterial isolation and this study showed isolation of six bacterial types (47 isolates) and also revealed that Gram positive bacteria were dominant, particularly Bacillus spp. and Staphylococcus aureus (Table 5 ) as mentioned by some studies $(16,22)$. More frequently mixed isolation from these specimens included Bacillus spp. with Enterococcus faecalis (Table 6).

The results of gizzard's specimens referred that these bacterial types may come from environment through contaminated food and water, then when passed to intestine definitely they spread to the other organs $(2,24)$.

Seven bacterial types (included 57 isolates) isolated from intestines of quail birds, Corynebacterium spp. and $E$. coli represented in a high percent (Table 7), also these two types isolated together more frequently as a mixed culture (Table 8). Other isolated bacteria from intestineswere involved Enterococcus faecalis, Staphyloc-occusaureus, Klebsiella pneumonia, Proteus spp. and Bacillus spp. These results accepted with the previous studies in the same line $(2,15,16,18,20,21)$, which refered that the intestinal bacteria may be the main source for contamination of other internal organs (21).

This study showed that Corynebacterium spp. were dominant in the organs of quail birds and this predominant may be explained by worldwide distribution of these bacteria during previous 2 decades and some studies related these bacteria with many infections in humans $(17,25,26)$.

On the other hand the Japanese quail are reported to be resistant to many diseases and in addition many bacterial isolates obtained from Japanese quail and their environment showed high resistance to multiple drugs with $100 \%$ (like isolates of E. coli) resistance observed against ampicillin/ cloxacillin, chloramphenicol, tetracycline, and cotrimoxazole and other antibiotics $(16,27,28,29)$, so that these birds may act as mechanical transporting for different bacterial species to humans and animals with the risky of transporting of resistance bacterial species.

\section{Acknowledgements}

The authors thank the College of Veterinary Medicine, University of Mosul for support. 


\section{References}

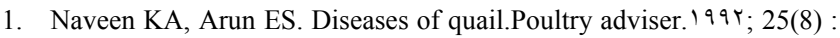
43-48.

2. Shane MS. Handbook on Poultry Diseaes.2ed.Ameri Soybean Associ. Singapore, 2005:p. 27,53-55, 114.

3. Mounteny GJ. Poultry products technology, second ed., The Avi pub.co. Inc. Westport Connecticut.1981:p. 67-69.

4. Abd El-Gawad AH, Hemid AEA, El-Wardany I. Alleviating the effect of some environmental stress factors on productive performance in japanesequail 1. growthperformance. W J AgriculSci 2008; 4 (5): 605611.

5. EI-Dangawy RA, NassarAM. Investigation on the nutritive value and micro-bialogical quality of wild quail carcasses.Nahrung 2001; 4 (5): 50-54.

6. AI-Nakhli HM. Occurrence of paratyphoid infection among japanesequails (Coturnixcoturnix japonica) in Saudi Arabia. Saudi J Bio Sci.2005; 12(1): 59-66.

7. Paulillo AC, Schmidt EM. Experiential vaccination against Newcastle disease in japanese quails (Coturnixcoturnix japonica): clinical and immunological param-eters. InterJPoultSci. 2009; 8(1):52-54

8. Edris AM, Shaltout FA, Arab WS. Bacterial evaluation of quail Meat.Benha Vet MedJ. r . ₹ ;16 (1):1-14

9. Quinn PJ, Markey BK, Carter ME, Donnelly WJC, Leonard FC. Veterinary Microbiology and Microbial Diseases.Blackwellpub Co.USA.2003 :p. $84-96$.

10. Koneman EW, Allen SD, Dowell VR, Janda WM,Sommers HM, Winn WC. Color Atlas of Diagnostic Microbiology $3^{\text {rd }}$ ed., Lippincott Co.1988:p. 87-92, 24, 115.

11. Quinn PJ, Carter ME, Markey B, Carter GR. Clinical Veterinary Microbiology $6^{\text {th }}$ ed. Mosby. Edinburgh, New-york.2004:p. 191-208.

12. Barrow GI, Feltham RKA. Cowan and Steel's manual for the identification of medical bacteria. University of Cambridge, Cambridge, United Kingdom. 2003:p.28-48.

13. ArunK Bhunia. Foodborne Microbial Pathogens. Springer Science+Busi-ness Media, LLC.2008: 126,197.

14. Ratnamohan N. The management of japanese quail and their use in virolo-gical research: A review. VetRes Comm. 1985; 9: 1-14

15. Mohamed HA. Proteus infection in quails in Assuit governorate.Assuit Vet Med J. 2004; 50(101): 196- 204
16. Roy P, Purushothaman V, Koteeswaran A, Dhillon AS. Isolation, Character-ization, and antimicrobial drug resistance pattern of Escherichia coliisolated from japanesequail and their environment. $\mathrm{J}$ Appl Poult Res. 2006;15:442-446.

17. Dhasarathan P, Uma GG, Rajkumar Seasonal variations in in microbial pop-ulation in sivakasi soil with reference to the influence of temperature. Pollu Res. 2006; 25(1): 114-118.

18. Lauková A, Michlovičová G. Enterococci isolated from japanesequails exposed to microgravity conditions and stability of their properties. ActaVet Brno.2009; 78:253-258.

19. Awad-Alla ME, Abdien HMF, Dessouki AA. Prevalence of bacteria and parasites in white ibis in Egypt. Vet Itali. 2010; 46 (3): 277-286.

20. Hong-lin W, Jun Y, Hua-bin S, Ling L, Di-yun A, Qing-ping L, Guoyuan W, Rong-rong Z, Lin Z. Isolation and identification of EnteropathogenicE. coli and Salmonella from quail and their drug sensitivity test. Vet Rec.2010;166:147-148.

21. Schmidt RE, Reavill DR, Phalen DN. Pathology of pet and aviary birds. A Blackwell Pub Com. $1^{\text {st }}$ ed. 2003:p.17-23,56-58, 74.

22. Burns KE, Otalora R, Glisson JR, Hofacre CL. Cellulitis in Japanese quail (Coturnixcoturnix japonica). Avian Dis. 2003; 47(1):211-214.

23. Thenmozhi $\mathrm{V}$, Malmarugan $\mathrm{S}$, Suresh $\mathrm{P}$, Jeyanthi $\mathrm{C}$. Isolation and identification of bacterial respiratory pathogensinjapanesequails. Indi J FieldVeterin. 2010; 6(1): 54- 56)

24. Thomas NJ, Hunter DB, Atkinson CT. Infectious Diseases of Wild Birds. Blackwell Pub. ${ }^{\text {st }}$ ed. 2007:P. 237-245, 265, 284.

25. Collins MD, Cummins CS. Genus Corynebacterium In: Sneath PHA, Mair NS, Sharpe ME, Holt JG.Bergey'sManual of Systematic Bacteriology. William \& Wilkins, Baltimore.1986:p.1293-1386.

26. Krech $\mathrm{T}$, Hollis DG.Corynebacterium and related organisms. In: Balows AB, Hausler WJ, Herrann KL, Isen-berg HD, Shadomy HJ. Manual of Clinical Micro-biology, $5^{\text {th }}$ ed. AmeriSoci Micro, Washington. D.C. 1991:p.277-286.

27. Nazer AH. Transmissible drug resistance in Escherichia coli isolated from poultry and their carcasses in Iran. Cornell Vet. 1980;70:365371.

28. Scioli C, Espostito S, Anzilotti G, Pavone A, Pennucci C. Transferable drug resistance in Escherichia coli isolated from antibiotic-fed chickens. Poult Sci. 1983; 62:382-384.

29. Barnes H J, Gross WB. Colibacillosis :in Diseases of Poultry. $10^{\text {th }}$ ed. B. W. Calneked. Mosby-Wolf PubLtd., London, UK. 1997 :p. 131139 